\title{
Entropy generation due to the mixed convection flow of MWCNT-MgO/water hybrid nanofluid in a vented complex shape cavity
}

\author{
Mahdi Benzema ${ }^{1, *}$, Youb Khaled Benkahla ${ }^{1}$, Ahlem Boudiaf ${ }^{1}$, Seif-Eddine Ouyahia ${ }^{1,2}$, and Mohammed El Ganaoui ${ }^{3}$ \\ ${ }^{1}$ Laboratory of Transport Phenomena, Faculty of Mechanical and Process Engineering, USTHB, B.P. 32, El-Alia Bab-Ezzouar, 16111 Algiers, \\ Algeria \\ ${ }^{2}$ Sonatrach - Direction Centrale Recherche et Développement. Avenue du 1er novembre - Boumerdes 35000 - Algérie \\ ${ }^{3}$ Université de Lorraine, LERMAB, IUT Longwy, 54400 Cosnes et Romain, France
}

\begin{abstract}
This paper reports a numerical study of mixed convection heat transfer with entropy generation in a vented complex shape cavity filled with MWCNT-MgO (15:85 vol \%) /water hybrid nanofluid. A hot source is placed at the mid potion of the inclined plate of the enclosure, while the rest of the rigid walls are adiabatic. A thermo-dependent correlations proposed by [12] for the dynamic viscosity and the thermal conductivity, especially developed for the considered fluid, are used. After validation of the model, the analysis has been done for a Reynolds numbers ranging from 10 to 600 and total nanoparticles volume fraction ranging from 0.0 to 0.02 using the finite volume method. The predicted results of streamlines, isotherms, isentropic lines, average Nusselt number, average entropy generation and average Bejan number are the main focus of interest in the present paper.
\end{abstract}

\section{Introduction}

In recent years, growing attention have been paid to use nanofluids due to its ability to enhance the heat transfer performances in various industrial applications, due to the significant increase in thermal conductivity of the resulting fluid. This new class of fluids refers to a liquid in which are suspended metallic or nonmetallic nano-sized materials. Hybrid nanofluids as a continuation of nanofluids, can be obtained by dispersing in base the fluid a composite nanopowder, or different kinds of nanoparticles.

The mixed convective flow in ventilated enclosure is present in many transport processes and engineering applications such as building ventilation, heat exchanger, cooling of electronic devices, solar energy storage etc. Due to practical interest of the mixed convection heat transfer, a number of works have been devoted to nanofluids flow within ventilated cavities such as [1-4]. However, few investigations have dealt with mixed convection flow of hybrid nanofluid in open cavities, which makes it a still fertile research area. These include the numerical investigations of [5-7]. It is worth mentioning that these studies involved cavities of regular geometries, while in practice, actual cavities are often in an irregular shapes. The aforementioned studies are based on the first law of thermodynamics which is not able to provide information on the quality of available energy. Entropy generation concept, which is based on the quantification of the amount of irreversibilities during a process, is crucial to study any thermal systems in order to achieve the optimal performances. Hence, entropy generation in the convective flow of pure and nanofluids has recently been the subject of great interest. However, very few studies have been devoted to analyze the second law of thermodynamics for open cavity problems such as that of [7-11].

The present study performs a numerical investigation into the mixed convection heat transfer characteristics and entropy generation rate within a vented complex shape cavity filled with MWCNT MgO (15:85vol \%)/water hybrid nanofluid. The simulations focus specifically on the effects of the Reynolds number and the total nanoparticles volume fraction on the streamlines, isotherms, isentropic lines, average Nusselt number, average entropy generation and Bejan number.

\section{Problem definition and mathematical formulation}


We consider a two-dimensional ventilated cavity of complex shape, subjected to a laminar and steady jet of MWCNT-MgO (15:85vol \%) /water hybrid nanofluid (see Fig.1). The latter enters the enclosure from the top at relatively cold temperature $\left(T=T_{C}\right)$, and is evacuated through an inlet port placed at the left end of the bottom wall. The width of both the openings $l$ is assumed as $0.2 \mathrm{~W}$. The width and height of the cavity denoted by $W$ and $H$ respectively are equals. An isothermal heat source of size $d$ $=0.5 \mathrm{~S}$ is placed at the mid potion of the inclined surface of the cavity (with an inclination angle of $45^{\circ}$ to the horizontal), while the rest of the rigid walls are thermally insulated.

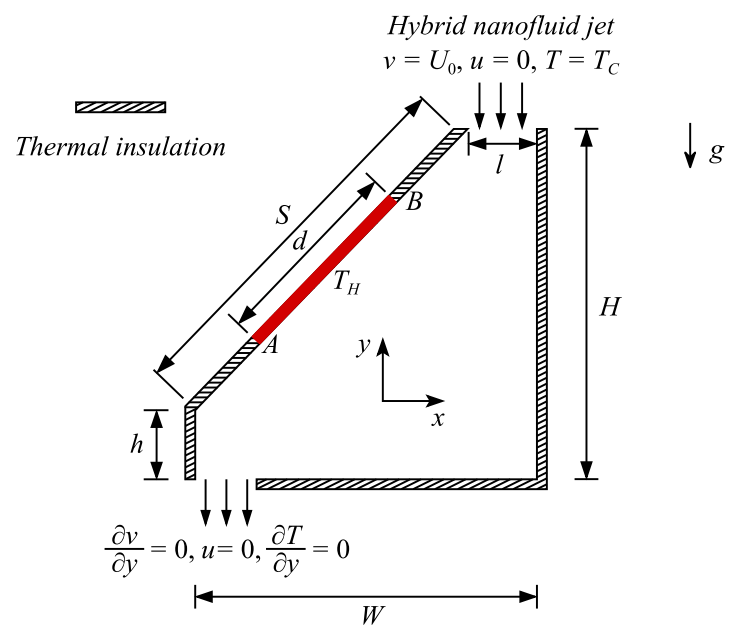

Fig. 1. Schematic diagram of the physical problem.

The fluid flow behavior is based on the single-phase nanofluid approach. It is assumed that the fluid is Newtonian incompressible with negligible effect of viscous dissipation. Considering these assumptions, the dimensionless governing equations, i.e., conservation of mass, momentum and energy, describing the flow under the Boussinesq approximation can be expressed as:

$$
\begin{gathered}
\nabla \cdot \vec{V}=0 \\
\vec{V} . \nabla \vec{V}=-\nabla P+\frac{\rho_{f}}{\rho_{h n f}} \frac{\mu_{h n f}}{\mu_{b f}} \frac{1}{R e} \nabla^{2} \vec{V}+\frac{(\rho \beta)_{h n f}}{\rho_{h n f} \beta_{b f}} R i \theta \\
\vec{V} . \nabla \theta=\frac{k_{h n f}}{k_{b f}} \frac{\left(\rho c_{p}\right)_{b f}}{\left(\rho c_{p}\right)_{h n f}} \frac{1}{\operatorname{RePr}} \nabla^{2} \theta
\end{gathered}
$$

Eqs. (1)-(3) are non-dimensionalized using the following reference values:

$$
\begin{gathered}
X=\frac{x}{H}, Y=\frac{y}{H}, U=\frac{u}{U_{0}}, V=\frac{v}{U_{0}}, P=\frac{p}{\rho_{h n f} U_{0}^{2}}, \theta=\frac{T-T_{C}}{T_{H}-T_{C}}, \\
\operatorname{Re}=\frac{\rho_{b f} U_{0} H}{\mu_{b f}}, P r=\frac{v_{b f}}{\alpha_{b f}} R i=\frac{g \beta_{b f} \Delta T H}{U_{0}^{2}}=\frac{G r}{\operatorname{Re}^{2}}
\end{gathered}
$$

In this study, the dynamic viscosity of the considered hybrid nanofluid $\mu_{h n f}$ and its thermal conductivity $k_{h n f \text {, }}$ are calculated using empirical correlations, proposed by Kasaeipoor et al. [12]:

$$
\begin{aligned}
\frac{\mu_{h n f}}{\mu_{b f}} & =1.027+0.2815 \varphi-0.00068 T-0.0761 \varphi^{2}-0.0086 \varphi T \\
& -1.732 e-05 T^{2}+0.0244 \varphi^{3}-0.00092 \varphi^{2} T+0.00018 \varphi T^{2} \\
\frac{k_{h n f}}{k_{b f}} & =0.9971+0.0788 \varphi+0.0048 T-0.108 \varphi^{2}+0.0078 \varphi T \\
& +0.0913 \varphi^{3}-0.0066 \varphi^{2} T-0.0258 \varphi^{4}+0.00201 \varphi^{3} T
\end{aligned}
$$

Where $\varphi$ is the total volume fraction (in percentage \%) of the two different types of nanoparticles dispersed in water which is calculated as: $\varphi=\varphi_{\mathrm{MWCNT}}+\varphi_{\mathrm{MgO}}$. It should be mentioned that the above correlations are valid for $0.25 \% \preccurlyeq \varphi \preccurlyeq 2 \%$ and $20^{\circ} \mathrm{C} \preccurlyeq \mathrm{T} \preccurlyeq 40{ }^{\circ} \mathrm{C}$. In general the properties of the hybrid nanofluid are evaluated via the following mixture law:

$$
\zeta_{\text {hnf }}=\left(1-\varphi_{M W C N T}-\varphi_{M g O}\right) \zeta_{b f}+\varphi_{M W C N T} \zeta_{M W C N T}+\varphi_{M g O} \zeta_{M g O}
$$

Where $\zeta$ stands for the following properties: the density $\rho_{h n f}$, the specific heat $\left(\rho \mathrm{c}_{p}\right)_{h n f}$ and the thermal expansion $(\rho \beta)_{h n f}$ of the hybrid nanofluid. Table 1 summarizes the values of the different parameters used to estimate the thermophysical properties of the hybrid nanofluid.

Table 1. Thermophysical properties water, MWCNT and $\mathrm{MgO}$ at $T=300 K[13-15]$.

\begin{tabular}{ccccc}
\cline { 2 - 5 } & $\begin{array}{c}\rho \\
\left(\mathrm{kg} \mathrm{m}^{-3}\right)\end{array}$ & $\begin{array}{c}\beta \times 10^{5} \\
\left(\mathrm{~K}^{-1}\right)\end{array}$ & $\begin{array}{c}\mathrm{C}_{\mathrm{p}} \\
\left(\mathrm{J} \mathrm{kg}^{-1} \mathrm{~K}^{-1}\right)\end{array}$ & $\begin{array}{c}\mathrm{K} \\
\left(\mathrm{W} \mathrm{m}^{-1} \mathrm{~K}^{-1}\right)\end{array}$ \\
\hline Water & 997.1 & 1.67 & 4179 & 0.613 \\
\hline MWCNT & 1600 & 4.20 & 796 & 3000 \\
\hline $\mathrm{MgO}$ & 3560 & 1.13 & 955 & 45 \\
\hline
\end{tabular}

Local and average Nusselt number on the heated surface could be given as follows:

$$
N u_{l}=-\left.\frac{k_{h n f}}{k_{b f}} \frac{\partial \theta}{\partial n}\right|_{\text {Heatsource }} \quad ; \quad N u_{m}=\frac{1}{\sqrt{2} H} \int_{A}^{B} N u_{l} d S
$$

where $n$ is the vector normal to the inclined active wall $\left(n=\sqrt{d X^{2}+d Y^{2}}\right)$. 
According to Bejan [16] and by using the dimensionless parameters introduces in Eqs. (4), the local dimensionless entropy generation $S_{g e n}$ from the various physical sources can be obtained as follows:

$$
\begin{aligned}
S_{g e n} & =S_{g e n}^{\prime} \frac{T_{0}^{2} H^{2}}{k_{b f}\left(T_{H}-T_{C}\right)^{2}}=\frac{k_{h n f}}{k_{b f}}\left[\left(\frac{\partial \theta}{\partial X}\right)^{2}+\left(\frac{\partial \theta}{\partial Y}\right)^{2}\right] \\
& +\chi \frac{\mu_{h n f}}{\mu_{b f}}\left\{2\left[\left(\frac{\partial U}{\partial X}\right)^{2}+\left(\frac{\partial V}{\partial Y}\right)^{2}\right]+\left(\frac{\partial V}{\partial X}+\frac{\partial U}{\partial Y}\right)^{2}\right\} \\
& =S_{g e n, h t}+S_{g e n, f f}
\end{aligned}
$$

where $S_{g e n, h t}$ and $S_{g e n, f}$ denote the entropy generation due to heat transfer and fluid friction respectively. In above equation $\chi=\mu_{b f} T_{0} U_{0}^{2} / k_{b f}\left(T_{H}-T_{C}\right)^{2} \quad$ is the irreversibility factor.

The dimensionless average entropy generation $S_{\text {gen,m }}$ is obtained through the integration of Eqs. (9) in the entire computational domain, as indicated in Eqs. (10):

$$
S_{g e n, m}=\frac{1}{\vartheta} \int_{\vartheta} S_{g e n} \partial \vartheta=S_{g e n, h t, m}+S_{g e n, f f, m}
$$

Further, the average Bejan number $B e_{a v g}$, is a dimensionless parameter defined as the ratio between the entropy generation due to heat transfer by the overall entropy generation. It is known that when $B e_{a v g}$ approaches to 1 , the irreversibly due to heat transfer predominates, while when $B e_{a v g}<0.5$ the fluid friction irreversibility becomes the dominant one. The latter is expressed as:

$$
B e_{a v g}=\frac{S_{g e n, h t, m}}{S_{g e n, h t, m}+S_{g e n, f f, m}}
$$

\section{Numerical procedure and CFD code validation}

The governing equations (Eqs. (1) - (3)) was discretized over a computational domain uniformly meshed $(\Delta X=\Delta Y)$ using the finite volume method. The hybrid scheme was selected for the space discretization of the diffusion and convection terms. To suppress the checkerboard pressure problem the SIMPLER algorithm was applied. The resulting linear equations were solved iteratively by the Alternating Direction Implicit (ADI) scheme, through the successive under relaxation procedure. The iterative process is repeated until reaching the convergence which is stopped by the following criterion: $\left\|\varsigma^{\sigma+1}-\varsigma^{\sigma}\right\| /\left\|\varsigma^{\sigma+1}\right\| \leq 10^{-5}$. Where $\varsigma$ refers to general variables $(U, V, P$ or $\theta)$ and $\sigma$ represents the iteration level. The inclined surface of the enclosure is approached with zigzag lines as stairs shape. Details of this approach can be found in Ref. [17-19]. In order to determine a grid independent solution, a grid convergence study is performed for $R i=1, \varphi=0.02$ and $R e=10$ and 600. As can be observed from the Fig. 2, the grid size of $151 \times 151$ is good enough to get an accurate computations.

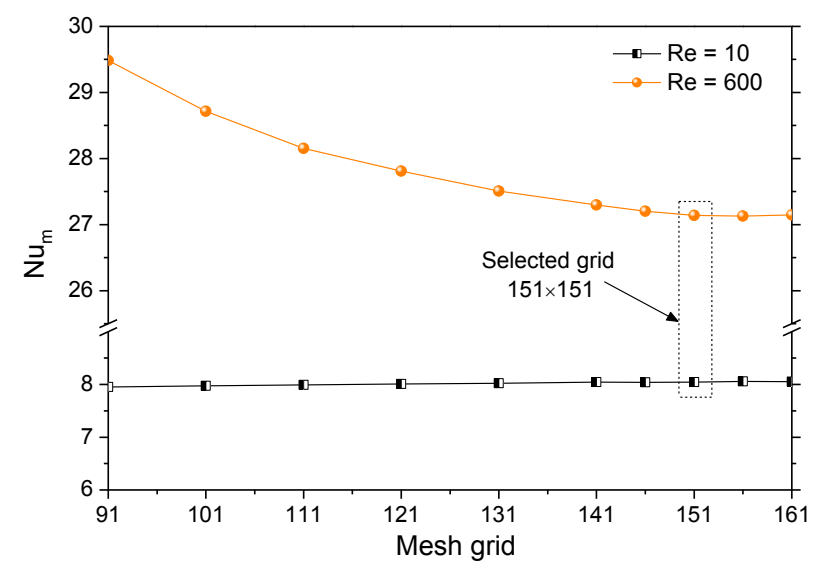

Fig 2. Cavity grid independence.

The numerical computations were performed on the basis of a homemade computer code written in FORTRAN programming language. The results were first validated for the mixed convection flow of $\mathrm{Al}_{2} \mathrm{O}_{3}$-water nanofluid in a ventilated square cavity for $R i=0, R e=500$ and pure fluid, as reported by Sortiji et al. [1]. Results are presented in Fig. 3 where a good agreement is observed.

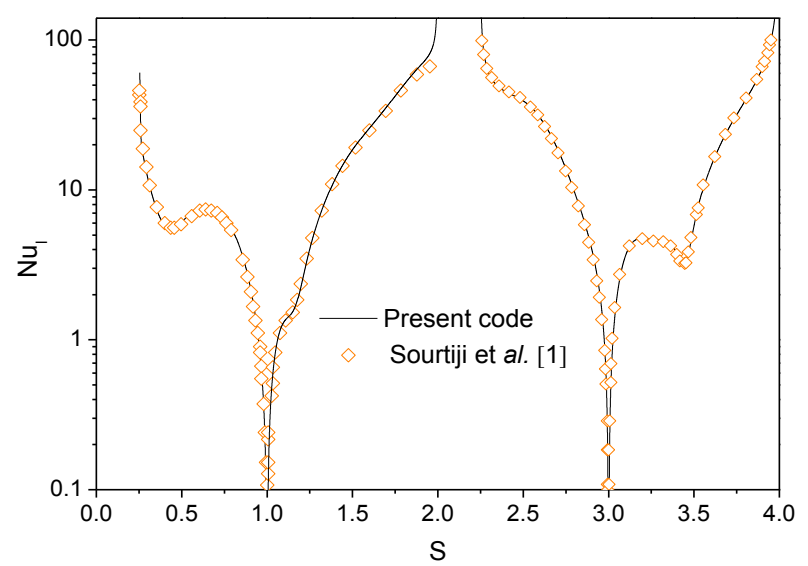

Fig. 3. Local Nusselt number according to the outlet opening position.

The second problem, which is selected to verify the performance of entropy generation part of the model, is from Mahmoudi et al. [18]. The results presented in Fig. 4, contrast the isentropic lines in a $\mathrm{Cu}$-water nanofluid filled trapezoidal cavity for $R a=10^{6}$ and $\varphi=0.0$. A close agreement is observed between both results.

Mahmoudi et al. [18] Present code 

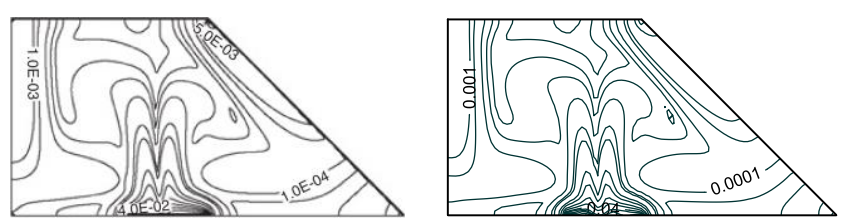

Fig. 4. Comparison of local entropy generation obtained by [18] and those of the present code.

\section{Results and discussion}

The mixed convection flow and heat transfer are investigated comprehensively using the entropy generation approaches. Particular efforts are focused on the effects of Reynolds number $(10 \preccurlyeq R e \preccurlyeq 600)$ and the total nanoparticle volume fraction $(0 \preccurlyeq \varphi \preccurlyeq 0.02)$ on the fluid flow, thermal field and local entropy generation as well as on the average Nusselt number, the average total entropy generation and the average Bejan number. In this study a base set of conditions was defined as $R i=1, \operatorname{Pr}=6.2$ and $\chi=10^{-2}$.

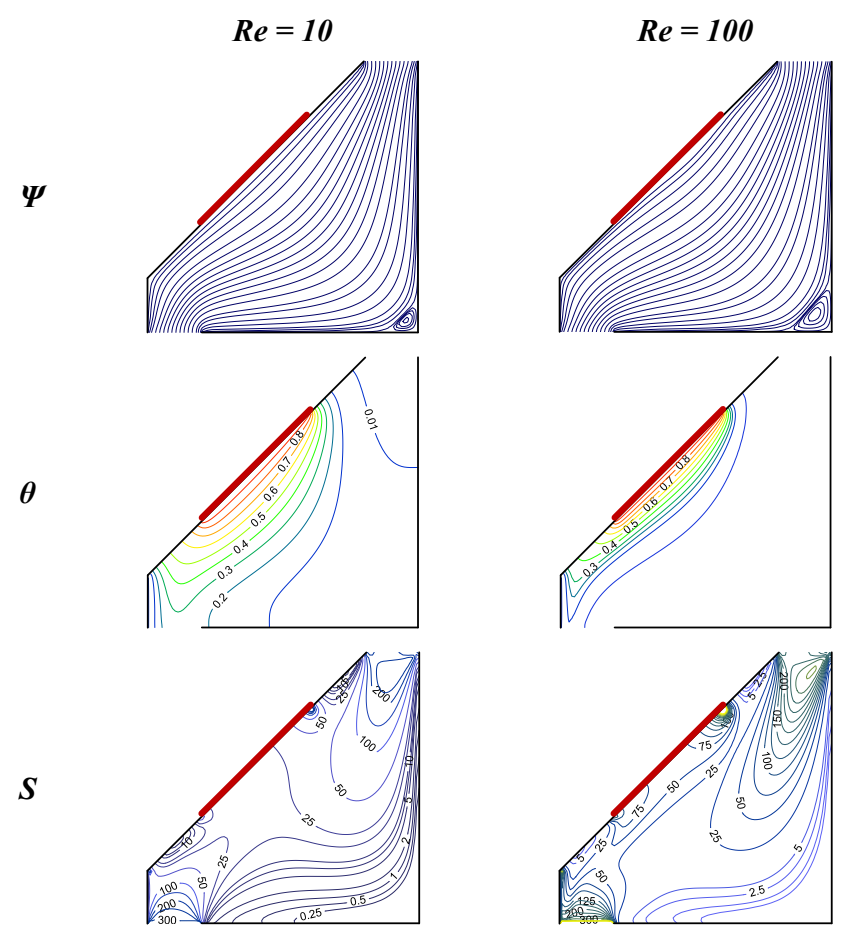

Figure 5 displays the effect of the Reynolds number on hydrodynamic, temperature and entropy generation fields at $\varphi=0.005$. It should be noted that for a fixed Richardson number value, the growth of the Reynolds number is related to an increase in Grashof number. It is observed that for $R e=10$, the main flow invades the major part of the cavity and a small counter-clockwise $(\mathrm{CCW})$ rotating cell is formed at the bottom right corner. This cell appears whatever the $R e$ values. The corresponding isotherms are almost uniformly distributed which points to the predominance of the conduction heat transfer mode. With the increase of $R e$ to 100 , the size of this cell increases slightly but the flow structure remains globally the same as that of the previous case. One can also observe the decrease in thickness of the thermal boundary layer along the active wall that is confirmed by an isotherms tightening near this wall. Note that in this case, a large part of the cavity is maintained isothermal and cold.

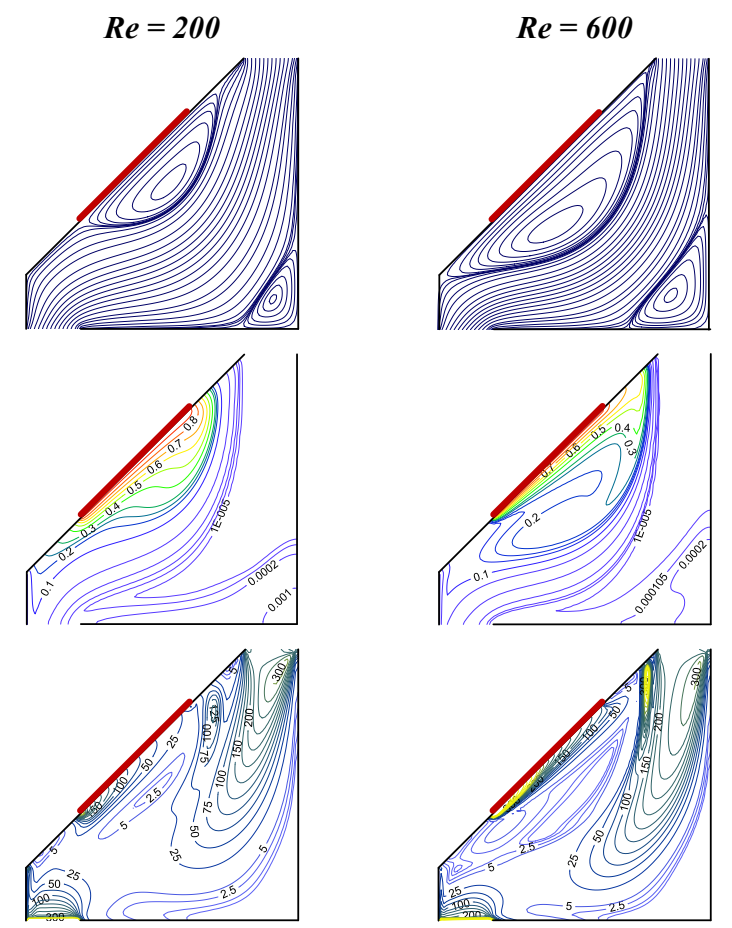

Fig. 5. The effect of Reynolds number on the streamlines, isotherms and isentropic lines $\varphi=0.005$.

At $R e=200$ the figure 5 illustrates a bi-cellular flow pattern characterized by the formation of a clockwise $(\mathrm{CW})$ rotating cell at the vicinity of the heater. Here, the buoyancy-driven flow starts to modify the flow structure. The isotherms contours regarding this case are more deformed, which indicates that the convection overlooks the conduction. One can be also see that the fluid temperature gradients are less marked close to the heat source in comparison with the case of $R e=100$. The weak cell formed in this region justifies this behavior. Here, the weak rotational motion of the $\mathrm{CW}$ justifies this behavior. When $R e=600$ the $\mathrm{CW}$ cell occupies a large portion of the enclosure and squeezes the forced flow. This is due to the intensification of the combined effects of the forced and natural convection modes. The temperature field shows a strong distortion of the isotherms indicating that the heat transfer is mostly governed by the convection mode. The corresponding thermal boundary layer close to the active surface becomes thinner. Moreover, it is seen 
that for all the cases of Fig. 5, the presence of a concentrator of entropy generation, formed by isentropic lines, which are connected from either side of the cavity entrance. This is partially due to the gradual increase of the cross section which causes important velocity gradients. This concentrator densifies and penetrates more region into the cavity with the increase of Re. Further, an important entropy generation occurs at the vicinity of the cavity exit due to the acceleration of hybrid nanofluid motion, and accumulation of heat in this region. One can also notice that with the appearance of the recirculation zone at $R e$ $=200$ and 600 , the heat source acts as a concentrator of entropy generation with a densification of isentropic lines as the fluid inertia increases. Figure 6 shows the variations of $N u_{m}$ with the Reynolds number and the total nanoparticle volume fraction. It is clear that for all $R e$ values, adding nanoparticles enhances the heat transfer due to the promotion of the fluid thermal conductivity. It can also be seen that with the increase in $R e$, the $N u_{m}$ takes a maximum at $R e=100$. In this case, the heat transfer enhancement is due to the growth of the fluid inertia that leads to strong velocity gradients close to the heat source, which reduces the thickness of thermal layer in this region (as seen in Fig.5).

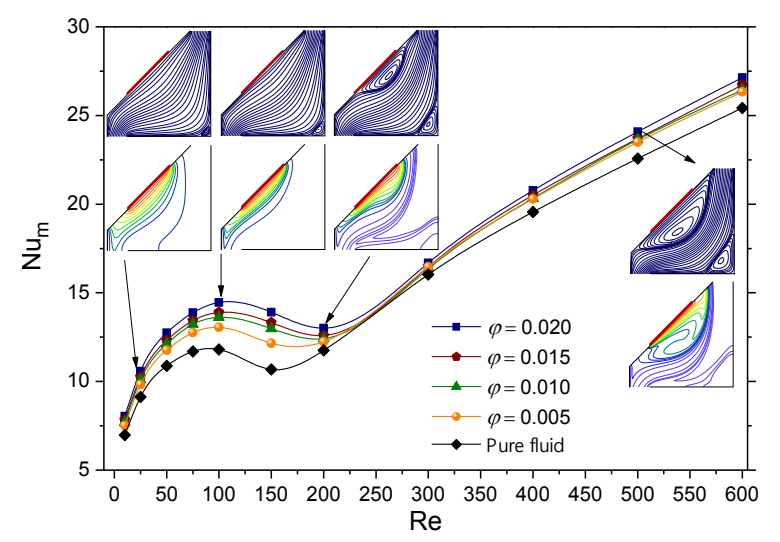

Fig. 6. Average Nusselt number versus Reynolds number for several values of $\varphi$.

Subsequently, the $N u_{m}$ decreases and takes a minimum depending of $\varphi$. In this case, the birth of the $\mathrm{CW}$ cell of low intensity, does not allow an efficient cooling of heat source. Beyond these minimums, $N u_{m}$ increases with the increase of Re due to the growth of both strength and size of the $\mathrm{CW}$ cell, which promotes the heat transfer. Figure 7 depicts the average entropy generation profile for the same treatment as that of the previous figure. It is seen that the increase of both the $\operatorname{Re}$ and $\varphi$ rising the total entropy generation rate. In fact, the increase of inertia forces intensifies the fluid friction effect and allows more important penetration of the heat in the cavity. Also, the increase in $\varphi$ leads to the growth of thermal conductivity and viscosity ratios appearing in the expression of $S_{\text {gen }}$ (eqs (9)).

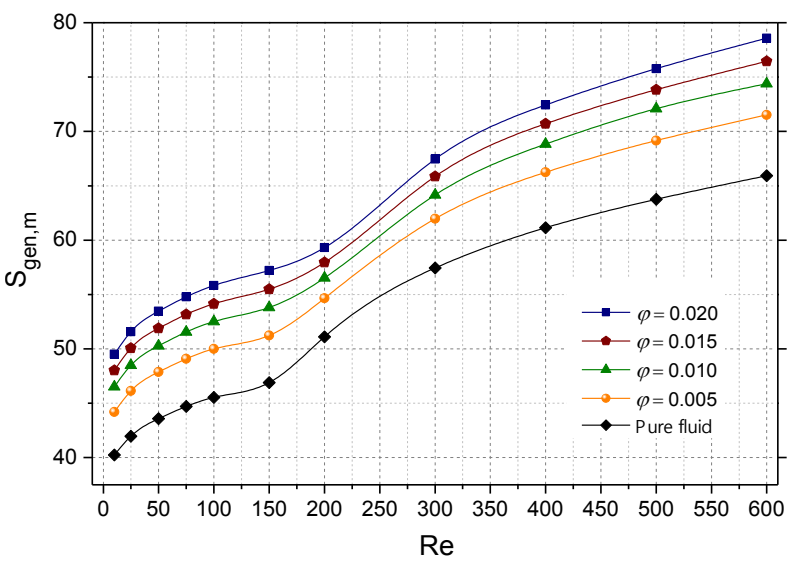

Fig. 7. Average Nusselt number versus Reynolds number for several values of $\varphi$.

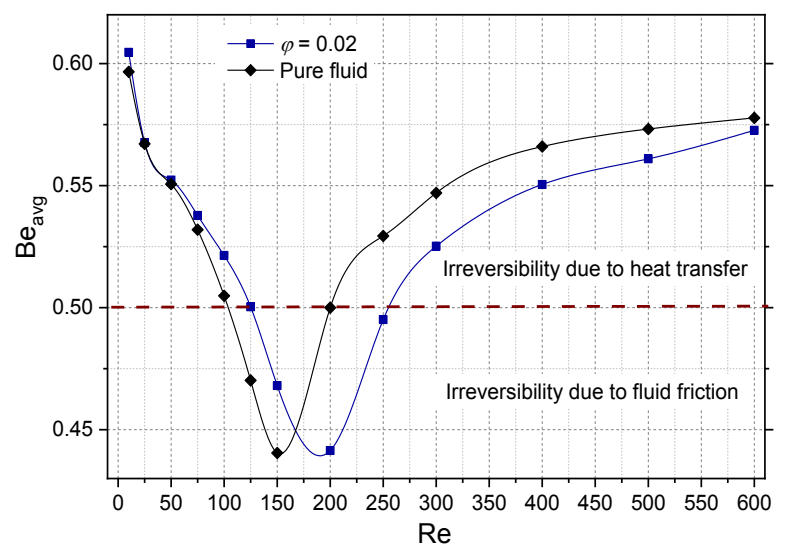

Fig. 8. Variation of average Bejan number with $\operatorname{Re}$ for $\varphi=0$ and 0.02 .

It can also be observed at high Reynolds number values. In this case, the high velocity of the fluid allows a greater distribution of the temperature within the cavity. It is to note that these $R e$ number ranges depend on the total nanoparticles concentration. In the lower region, corresponding to an intermediate Reynolds number range, the $B e_{\text {avg }}<0.5$, showing the domination of fluid friction irreversibility. In these cases the increase of fluid inertia leads to high velocity gradients which do not allow a significant penetration of the heat into the cavity.

\section{Conclusions}

Mixed convection heat transfer with entropy generation in a vented complex shape cavity crossed by MWCNT $-\mathrm{MgO} /$ water hybrid nanofluid has been numerically analyzed. Thermo-dependent correlations proposed by [12] to estimate the dynamic viscosity and the thermal conductivity of the considered hybrid nanofluid are employed. The simulations have been carried out for wide range of Reynolds number and total nanoparticle volume fraction. The obtained results show a complex flow pattern which is sensitive to the fluid 
inertia. Thus, the increase in Reynolds number makes the fluid flow patter from a unicellular to a bicellular structure. For all $R e$ values, adding nanoparticles enhances the heat transfer rate. Also, the increase of $R e$ to 100 promotes the heat transfer. Beyond this $R e$ value the $\mathrm{Nu}_{\mathrm{m}}$ decreases up to a minimum and then rises. The average entropy generation increases with $R e$ and $\varphi$. Finally, the $B e_{a v g}$ variations show the predominance of each irreversibility sources depends on the Reynolds number ranges.

\section{References}

1. E. Sourtiji, D. D. Ganji, and S. F. Hosseinizadeh, Powder Technol. 262, 11 (2014).

2. A. Kasaeipoor, B. Ghasemi, and S. M. Aminossadati, Int. J. Therm. Sci. 94, 11 (2015).

3. A. A. Mehrizi, M. Farhadi, H. H. Afroozi, K. Sedighi, and A. A. R. Darz, Int. Commun. Heat Mass Transf. 39, 9 (2012).

4. M. Shahi, A. H. Mahmoudi, and F. Talebi, Int. Commun. Heat Mass Transf. 37, 13 (2010).

5. K. Kalidasan, R. Velkennedy, and P. R. Kanna, J. Mol. Liq. 246, 8 (2017).

6. K. Kalidasan and P. R. Kanna, Int. Commun. Heat Mass Transf. 81, 8 (2017).

7. S. Hussain, S. E. Ahmed, and T. Akbar, Int. J. Heat Mass Transf. 114, 13 (2017).

8. Z. Mehrez, A. El, A. Belghith, and P. Le, $A d v$. POWDER Technol. 26, 14 (2015).

9. Z. Mehrez, E. Cafsi, A. Belghith, and P. Le, J. Magn. Magn. Mater. 374, 11 (2015).

10. A. A. A. A. Al-Rashed et al., Int. J. Mech. Sci. 135, 14 (2018).

11. F. Zamzari, Z. Mehrez, A. El Cafsi, A. Belghith, and P. Le Quéré, J. Hydrodyn. 29, 15 (2017).

12. A. Kasaeipoor, E. H. Malekshah, and L. Kolsi, Powder Technol. 322, 15 (2017).

13. R. Davarnejad and M. Jamshidzadeh, Eng. Sci. Technol. , an Int. J. 18, 7 (2015).

14. K. Kalidasan and P. R. Kanna, J. Taiwan Inst. Chem. Eng. 65, 10 (2016).

15. D. K. Smith and H. R. Leider, J. Appl. Cryst. 246, 4 (1968).

16. A. Bejan, J. Heat Transfer, 101, 8 (1979).

17. M. Benzema, Y. K. Benkahla, N. Labsi, E. Brunier, and S. Ouyahia, Arab. J. Sci. Eng., 42, 12 (2017).

18. H. A. Mahmoudi, I. Pop, M. Shahi, and F. Talebi, Comput. FLUIDS. 72, 17 (2013).

19. Ouyahia, S.-E.; Benkahla, Y.K.; Labsi, N, Arab. J. Sci. Eng. 41, 15 (2016). 